smaller in diameter and much longer in the length.

When the clot which has been produced by the mixture of CSF and blood is inocuated subdurally in the dog, a cyst encapsulated with the membrane is formed within one week. Histological examination of the capsule demonstrates that the external membrane consists of sinusoidal layer and fibrous layer and internal membrane is formed by a fibrous layer. These findings are comparable to those usually found in human cases.

When clot which has been formed in the existenec of CSF is inoculated subcutaneously, it grows gradually to reach its maximal size after two weeks, then thereafter the size of subcutaneous tumor is reduced. Again, histological findings of the capsule are quite similar to those seen in human chronic subdural hematoma.

When the surface of a regular clot is coated by fibrin membrane and inoculated subcutaneously a similar encapsulated cyst is formed.

In conclusion, it is assumed that fibrin membrane, particularly fibrin which is formed in the existence of CSF, plays an important role in the formation of chronic subdural hematoma.

\title{
n-2. Chronic Extradural Hematoma
}

Yutaka Inaba, Hiroshi Hata, Shigeru Toyota, Shunpei Takahashi,

Yasuyoshi Kayama, Akira Kamisasa, Yutaka HoshII

and Takashi MORIYAMA

Dept. of Neurosurgery, School of Medicine, Tokyo Medical and Dental University

\section{Discussion to $\mathbf{n - 2}$. \\ Extradural Hematoma with Prolonged Course}

\author{
Kenichiro HIGashI \\ Second Surgical Department, Yamaguchi University Medical School
}

Although it has been thought that chronic extradural hematoma having a long period before its disclosure was a relatively rare occasion, we experienced seven such cases which was operated on after more than 3 weeks following the head injury. These cases correspond to $13.3 \%$ of 52 extradural hematomas which have so far been operated on in our clinic. Therefore, we believe that the occurrence of extradural hematoma with a prolonged course is not so infrequent since cerebral angiography has become a prvalent diagnostic technique in recent years.

In only one out of these 7 cases, hematoma was found out unexpectedly at 